\title{
Knowledge, Machine Learning and Atrial Fibrillation: More Ingredients for a Tastier Cocktail
}

\author{
Tomas Teijeiro
}

\author{
Embedded Systems Laboratory (ESL), EPFL, Lausanne, Switzerland
}

\begin{abstract}
Fifty years after the publication of the first algorithms for the automatic detection of Atrial Fibrillation (AF), this cardiac condition is still the most studied from the computer science and engineering perspectives. Machine learning techniques are widely applied to a variety of problems, including detection, characterization, prediction and simulation, in general with promising results. In the last years, the Big Data + Deep Learning binomial is getting most of the attention in academia and industry, but on many occasions this approach fails on capitalizing all the knowledge acquired in previous decades of research.

This article, written as a companion to the keynote with the same title presented in the CinC 2020 conference, tries to illustrate the importance of exploiting expert knowledge and classical approaches in synergy with the most advanced deep learning methods, which by themselves have fundamental limitations. The discussion is built around the AF detection problem and the conclusions extracted from the Physionet/CinC Challenge 2017, but the main points can be relevant in other problems for which humans have a better answer than computers, and this answer can be described.
\end{abstract}

\section{Introduction}

The application of computer science to study atrial fibrillation has been explored for more than 50 years. Probably one of the first references is the work by Gersch et. al. [1], aimed at AF classification. Interestingly, this is still the most studied problem nowadays, even if that first paper reported a classification accuracy of $100 \%$.

$\mathrm{AF}$ is one of the cardiac conditions with a simpler diagnosis procedure based on the ECG signal, that can be summarized in three conditions [2]:

- Irregularly irregular heart rate.

- Absence of P waves.

- Presence of $f$ waves.

This apparently simple description makes it particularly suitable for formalization, and together with the high per- formance obtained by the first proposed methods [3] has led sometimes to a consideration of "easy problem".

However, this is just the tip of the iceberg, and a simple bibliographic query ${ }^{1}$ shows that just in the last 20 years, almost 275000 new publications have arisen on the topic of $\mathrm{AF}$ detection. It is important to note that under this generic naming there is a wide range of problems conditioned on many variables, and with incomparable difficulties. We may target different AF types (paroxysmal, persistent, permanent, ...), faced just with normal sinus rhythms or with other possible concurrent arrhythmias, using different signals for detection (ECG, PPG, respiration, ... ), in a shortterm or a continuous monitoring scenario, targeting an embedded implementation in a wearable device or a serveroriented deployment, etc [2].

Moreover, there are plenty of other problems related to AF management beyond detection, and that are attracting a lot of interest from the computer science community. Here we can mention the extraction and characterization of $f$ waves, that have been found to be extremely valuable for the prediction of treatment outcomes [4] or the prognosis of patients undergoing catheter ablation [5] in the context of personalized interventions. Other interesting topics are the development of AF simulation models [2], patient risk stratification based on the electronic health record [6], or medication management [6].

Regarding the computational techniques used to tackle these problems, the evolution seems to be guided by the availability of data. Initially, efforts focus on finding parameters that show a statistically significant variation during the target condition [2]. The search is limited to a few parameters that have a supporting clinical hypothesis, and the validation is done on a small population from the same hospital or health centre (usually less than 20 subjects). Then, when more data becomes available, ideally in a public fashion, there is an explosion of explorative approaches trying to exploit all the available data with sophisticated methods, focusing on optimizing a performance metric. This is the stage in which we can find almost any combination of features, preprocessing methods, and machine

\footnotetext{
${ }^{1}$ Search in Google Scholar with query ("atrial fibrillation" detection)
} 
learning algorithms, curiously most of them claiming their superiority over the others. Finally, since we are in the Deep Learning era, this technique is nowadays the culmination of this exploration, receiving most of the academic interest even if in practice it rarely proved an advantage over more classical approaches $[2,7]$.

In the following sections, we will motivate the need to consider formalized expert knowledge as a key part of machine learning-based methods for AF management, taking as a reference the AF detection problem on ECG signals. We will illustrate how pure data-driven approaches based on deep learning face fundamental limitations, particularly with small and medium-size datasets, and how hybrid approaches integrating domain-specific knowledge can provide advantages not only in terms of model interpretability, but also if we just focus on model performance.

\section{Intrinsic limitations of data-driven ap- proaches: A toy example}

To illustrate the fundamental downside of approaches that attempt to build models of cardiac events solely from data, we performed a simple experiment in which we tried to solve the following problem:

Classification Problem: Given a single-lead ECG segment of 30 seconds, and its derived $R R$ sequence $\left\{R R_{1}, \ldots, R R_{n}\right\}$ measured in milliseconds, the segment is classified as positive if and only if:

$$
\begin{gathered}
\exists i \in\{1, \ldots, n-2\} \mid \max \left(R R_{i}, R R_{i+1}, R R_{i+2}\right)< \\
500 \vee \min \left(R R_{i}, R R_{i+1}, R R_{i+2}\right)>1000 .
\end{gathered}
$$

According to this definition, any ECG segment can be unambiguously classified as positive or not, with perfect class separability. The definition may look utterly arbitrary, but it is indeed similar to the type of rules we can find in current clinical guidelines [8]. In this case, as a classification problem it is completely naive, but it requires to perform four basic operations on the input signal:

1. Detection of QRS complexes.

2. Differentiation to extract the RR series.

3. Counting up to 3 .

4. Performing a logical OR operation.

We tried to solve this toy problem with a neural network architecture that has demonstrated good performance in arrhythmia detection from the raw ECG [9], and using the MIT-BIH Arrhythmia database as source data [10]. The 46 recordings containing the MLII lead were selected and split into two groups of 37 and 9 for training/testing. Then, each recording was cut into segments of 30 seconds, using a sliding window with different values of overlap to assess the importance of the data size. Specifically, we studied overlaps of 20,25, and 29 seconds between consecutive

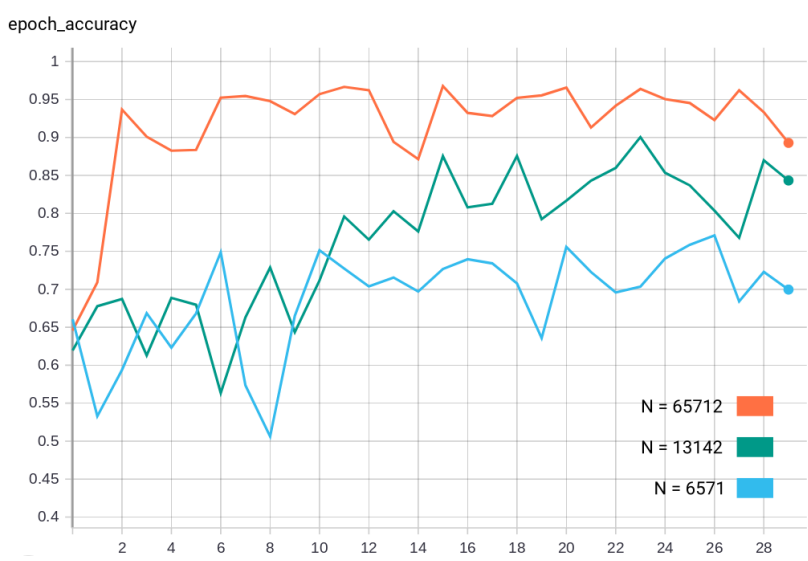

Figure 1: Learning curve of the neural network on a toy example problem.

segments, resulting in training set sizes of 6571, 13142 and 65712 samples, respectively. A binary label was assigned to each sample according to equation (1).

The prevalence of the positive label both in the training and test sets was around $25 \%$, which is a not so high unbalance for this type of problems and, therefore, no specific actions were taken to correct this. For training, we used the same optimizer and hyperparameter values described in [9].

Figure 1 shows the test set accuracy evolution for 30 epochs, considering different training sizes $N$. We can see that for $N=6571$ the accuracy is even lower than what would be achieved by a classifier giving always a negative prediction, but for $N=13142$ there is some effective learning, with a convergence between $80 \%$ and $85 \%$. For $N=65712$ the convergence is much faster to an accuracy range between $90 \%$ and $95 \%$, and if we use the F1 score to take into account the class unbalance the value is around 0.82 . We believe this is still a quite remarkable result considering that the network has been trained exclusively with the raw ECG, but it illustrates the difficulties and the large amounts of samples required to fit some basic quantitative conditions that usually guide humans when they perform such sort of classification tasks.

If the classification errors of the final model are considered, we can see that in this particular case the main difficulty relies on learning the rule of "three consecutive $R R$ intervals". Figure 2a shows a segment wrongly classified as negative, probably because it has exactly 3 consecutive RR intervals under the $500 \mathrm{~ms}$ threshold. On the other side, Figure $2 b$ shows an example of a false positive. It is a segment with multiple ectopic beats, leading to many RR intervals under $500 \mathrm{~ms}$, but these are isolated. Interestingly, even if the labels distribution in the training set is biased towards the negative class $(75 \%)$, most of the classification errors are false positives (1536 vs 177 false negatives). 


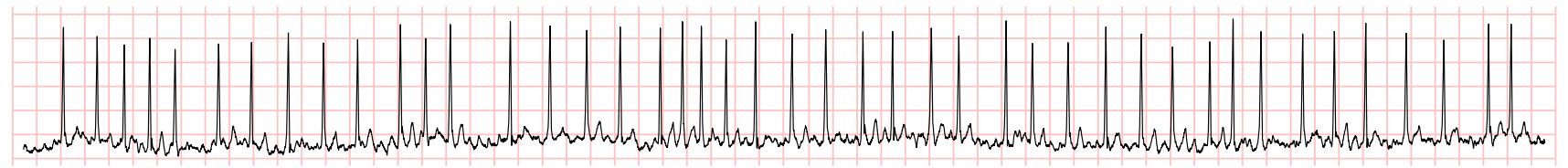

(a) False negative example.

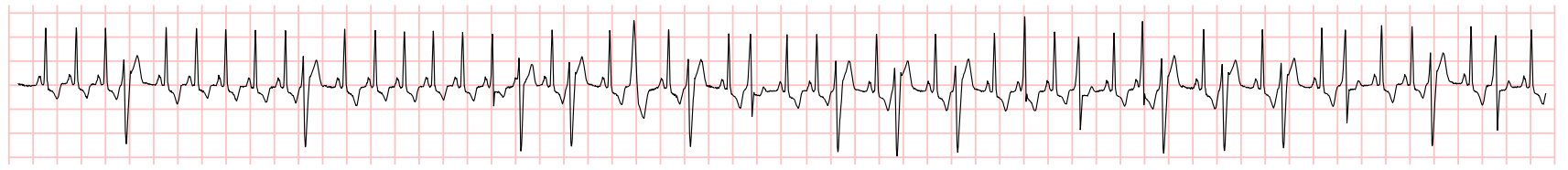

(b) False positive example.

Figure 2: Illustrative misclassified examples by the neural network.

\section{The importance of hybrid strategies: Lessons from the Physionet Challenge 2017}

The Physionet/CinC challenge 2017 is the latest public testbed for the evaluation of algorithms for AF detection in short single-lead ECG recordings. Algorithms should classify segments of 30 seconds duration in one of four classes (normal rhythm, AF, other rhythm, or noise) using a training set with 8528 recordings, while the evaluation was performed by the challenge organizers on a hidden test set with 3658 recordings.

Figure 3 shows the performance obtained by the top-25 algorithms in the follow-up stage of the Challenge [11], grouped by three categories:

- Classical ML: Solutions relying on handcrafted features linked to expert knowledge, and a classical machine learning model (typically ensembles of decision trees via gradient boosting or random forest, or support vector machines).

- Pure DL: Neural network models (typically convolutional or recurrent neural network architectures) trained directly on the raw ECG signal.

- Hybrid with DL: Hybrid solutions that combine Classical ML and DL models using handcrafted features linked to expert knowledge.

The first conclusion we can get is that machine learning has an advantage over pure knowledge-based systems and single or multivariate statistical methods (no such methods were proposed, or achieved the top-25 rank), particularly when there are no consistent definitions of the target labels. The ability to automatically fit a decision function can help not only to optimize the score on a particular target dataset, but also to highlight the inconsistencies among different human labelers [12], potentially leading to an improved definition of the target conditions.

On the other hand, it is interesting to see that Pure DL approaches are all clustered around the same positions and with minimum score differences among them. This suggests that the network type and architecture, the employed

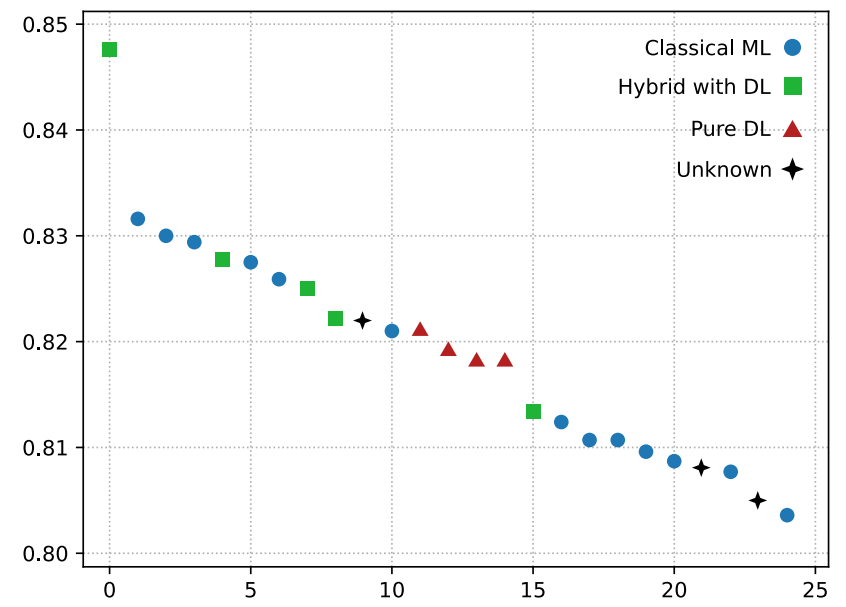

Figure 3: Performance of the top-25 algorithms in the Physionet/CinC Challenge 2017.

optimizer or the selected hyperparameters are of very little importance, and the main limitation seems to come from not exploiting the domain knowledge embedded in the features employed by other approaches. These features are mostly encoding the morphological and rhythm information that is known to be related to AF, and for this information to arise just from the raw ECG it would probably be necessary a dataset several orders of magnitude larger [13]. Even in this case, it is not clear whether this would happen, since usually label quality drops as dataset size increases [7], and this may hinder the discovery of edge conditions.

Finally, regarding the best-performing solution [14], which I was lucky to participate in its development, we can highlight two main aspects that were not explored in any of the other approaches: 1) the interpretation of the ECG in multiple abstraction levels prior to the feature extraction, and 2) the partial relabeling of the training set to improve its internal consistency. The interpretation is done in a pure knowledge-based fashion, and it ends up describ- 
ing the ECG as a sequence of waves, and also as a sequence of rhythms. This makes it possible to create features that are more abstract, such as "proportion of the record length in which the patient showed a non-regular rhythm”. Hypothetically, if this interpretation is robust enough then the final classification should be direct without requiring any machine learning. However, the absence of explicit criteria for deciding the target class based on the pathophysiological description of a record, along with the inconsistencies found among the labels in the training set, made it essential to rely on machine learning for fitting these underlying criteria.

As for the relabeling of the training set, it demonstrates the importance of having internal consistency between features and labels, which can be summarized as follows: "If your features don't grasp the information required to distinguish two examples, it is better that they have the same label". The strategy followed to relabel was based on the errors made by the classifier during cross-validation, and following the assumption that if an error is due to a mislabeled example, changing the label would improve the classification performance for two classes. On the contrary, if the error is due to an outlier, changing the label would only improve the performance of that class, but penalize the new one.

\section{Conclusion}

It is uncertain if in another 50 years atrial fibrillation will continue to pose unsolved challenges in computer science and engineering. However, what seems likely is that machine learning will be behind the solution to many of the problems that will be crossed off the initial target list. Nevertheless, the human cardiovascular system will continue to behave in exactly the same way as it does today, and as it has for the past 150000 years. Thus, all the effort we devote to formalizing the available knowledge and apply it to problem-solving will result in more robust, reliable, interpretable, and trustworthy systems.

\section{Acknowledgments}

This work has been supported by the H2020 DeepHealth Project (GA No. 825111).

\section{References}

[1] Gersch W, Eddy DM, Dong E. Cardiac arrhythmia classification: A heart-beat interval-Markov chain approach. Computers and Biomedical Research 1970;3(4):385-392. ISSN 00104809.

[2] Sörnmo L (ed.). Atrial Fibrillation from an Engineering
Perspective. Series in BioEngineering. Cham: Springer International Publishing, 2018. ISBN 978-3-319-68513-7.

[3] Moody G, Mark R. A new method for detecting atrial fibrillation using R-R intervals. Computers in Cardiology 1983; 227-230.

[4] Bollmann A, Kanuru NK, McTeague KK, Walter PF, DeLurgio DB, Langberg JJ. Frequency analysis of human atrial fibrillation using the surface electrocardiogram and its response to ibutilide. American Journal of Cardiology 1998;81(12):1439-1445. ISSN 00029149.

[5] Alcaraz R, Hornero F, Rieta JJ. Electrocardiographic Spectral Features for Long-Term Outcome Prognosis of Atrial Fibrillation Catheter Ablation. Annals of Biomedical Engineering 2016;44(11):3307-3318. ISSN 15739686.

[6] Siontis KC, Yao X, Pirruccello JP, Philippakis AA, Noseworthy PA. How Will Machine Learning Inform the Clinical Care of Atrial Fibrillation? Circulation Research 2020; 127(1):155-169. ISSN 0009-7330.

[7] Clifford GD. The Future AI in Healthcare: A Tsunami of False Alarms or a Product of Experts? arXiv Preprint 2020; URL http: / / arxiv.org/abs/2007.10502.

[8] Simpson RF, Langtree J, Mitchell AR. Ectopic beats: How many count? EMJ Cardiology 2017;

[9] Warrick P, Homsi MN. Cardiac arrhythmia detection from ecg combining convolutional and long short-term memory networks. In Computing in Cardiology, volume 44. IEEE Computer Society, 2017; 1-4.

[10] Moody GB, Mark RG. The impact of the MIT-BIH arrhythmia database, 2001.

[11] Physionet challenge 2017: Full test set scores for followup entries released, 2018. URL https://groups. google.com/g/physionet-challenges/c/ qA2iUfQmRtc/m/OHQP ImxEAwAJ.

[12] Clifford GD, Liu C, Moody B, Lehman LH, Silva I, Li Q, Johnson AE, Mark RG. AF classification from a short single lead ECG recording: The PhysioNet/computing in cardiology challenge 2017. In Computing in Cardiology, volume 44. IEEE Computer Society, 2017; 1-4.

[13] Attia ZI, Noseworthy PA, Lopez-Jimenez F, Asirvatham SJ, Deshmukh AJ, Gersh BJ, Carter RE, Yao X, Rabinstein AA, Erickson BJ, Kapa S, Friedman PA. An artificial intelligence-enabled ECG algorithm for the identification of patients with atrial fibrillation during sinus rhythm: a retrospective analysis of outcome prediction. The Lancet 2019; 394(10201):861-867. ISSN 01406736.

[14] Teijeiro T, García CA, Castro D, Félix P. Abductive reasoning as the basis to reproduce expert criteria in ECG Atrial Fibrillation identification. Physiological Measurement 2018;39(8).

Address for correspondence:

Tomas Teijeiro

ELG 136 (Building ELG), Station 11, EPFL

CH-1015 Lausanne. Switzerland

tomas.teijeiro@epfl.ch 